\title{
Performance evaluation of vehicle front structure in crash energy management using lumped mass spring system
}

Sunday M Ofochebe ${ }^{1 *}$, Chigbogu G Ozoegwu ${ }^{1}$ and Samuel O Enibe ${ }^{2}$

\author{
* Correspondence: \\ aa_zee2@yahoo.com \\ ${ }^{1}$ Department of Mechanical \\ Engineering, Nnamdi Azikiwe \\ University, Awka, Nigeria \\ Full list of author information is \\ available at the end of the article
}

\begin{abstract}
Background: The design for vehicle structural crashworthiness which ensures that components of desired crash performance characteristics are used in product manufacturing essentially involves the evaluation of the energy absorption potentials of the structures using suitable computation method. Due to unresolved difficulties in achieving detailed results through the existing methods researchers seek for more alternative computation methods. Although previous efforts in this regard are quite significant, yet some concerns still exist on accuracy or computational efficiency achievable through the conventional methods.

Method: The lumped mass spring (LMS) method is applied in the present study. Some new steps were introduced in the basic procedure to improve the accuracy and computational efficiency of the method. A new dynamic stiffness formula is written in terms of the specific energy absorption indices of the structural components. The new procedure allowed for standard state-space formulation of the crash problem.
\end{abstract}

Results: The performance of the new simulation approach is tested for a typical vehicle structure in two possible orientations called normal mode and reversed mode. The results obtained for the impact problem in normal structural mode show that a desirable energy absorption pattern of $45 \%, 25 \%$ and $20 \%$ of the total impact energy could be achieved through plastic deformation of the front frame, sheet metal and torque box respectively. Testing the impact system in reversed structural mode results in a rather poor energy absorption pattern in which $2.5 \%, 50 \%$ and $43 \%$ of the total impact energy were absorbed through deformation of the front frame, sheet metal and torque box respectively, showing that unreasonably high percentage of the total impact energy is transmitted to the interior structures.

Conclusion: The effort to quantify the energy absorbed by major vehicle front structure in both desirable and undesirable crash responses, and the computational efficiency achieved through the present method could help to enhance decision process during assessment of the components or prototype. It is found that good crash performance may be guaranteed by ensuring sufficiently high (up to 65\%) contribution to the energy absorption scheme through the deformation of the foremost structures which includes the front frame and the sheet metal.

Keywords: Crash energy; Lumped mass; Performance evaluation; Load zone; Deformation; Crashworthiness

\section{Springer}

@ 2015 Ofochebe et al.; licensee Springer. This is an Open Access article distributed under the terms of the Creative Commons Attribution License (http://creativecommons.org/licenses/by/4.0), which permits unrestricted use, distribution, and reproduction in any medium, provided the original work is properly credited. 


\section{Background}

Mechanical systems can be formulated into analytical models by integrating the inertial, stiffness and energy absorption/dissipation properties of the real system in an equivalent arrangement of solid mass(es), spring(s) and/or damper(s) known as lumped mass spring (LMS) system. The development of such an analytical method has been of great importance to engineering and science owing to its relative simplicity in resolving certain research problems about the real world system it represent. Of particular importance is its significant success in vehicle crash modeling which currently undermines any other alternative analytical approach. The viability of LMS system approach in resolving nonlinear crash problems is established by the possibility of updating the input variables in the dynamic states using time-stepping numerical integration technique which usually leads to a valid approximate solution. Lumped mass spring system approach provides holistic and handy information on the dynamics of the vehicle impact based on the underlying physics of the impact system; assuming strong and undeformable components to be rigid with concentrated masses contributing significantly to the distribution of inertia forces and transmission of impact energy, while the compliant (deformable) structural components are considered to have uniformly distributed masses contributing substantially to the dynamic resistance and energy absorption sequence. Interpreting such concrete information during prototype or component assessment is usually straightforward and requires no further averaging or integration of any sub-critical (nodal) information. However, existing reports show that achieving an acceptable accuracy via LMS approach in vehicle impact problems involves rigorous characterization of the complex elastic-plastic motion observed during structural deformation under dynamic impact condition. The necessary tasks regrettably present some unique challenges, ranging from the cost of achieving a reliable input data to the governing equation of motion to the computational difficulties in describing such input data in the dynamic state. These facts pose a perpetual hindrance to the application of the highly rated LMS system approach in precise evaluation of energy absorption potential of vehicle structure during impact. Nevertheless very few successful efforts to obtain a reliable estimate of vehicle component crush behavior using well organized impact experiment reported in literature have been of great help to researchers in expanding the scope research in this important subject. For instance, Balike successfully utilized the static crush test data (i.e. force-deformation characteristics obtained at quasi-static condition) recorded for various components of a typical framed car [1] for evaluating the performance of under-ride guard in idealized collision involving a lightweight passenger car and a heavy truck [2]. In other related works, the results of the crash calibration test of a standard Ford Fiesta [3] have been used to test the performance of linear visco-elastic models like the Maxwell model [4], Kelvin model [4,5], and auto-regressive models [5] in correlating real vehicle crash responses. The idea of assuming a perfect rigid mass in appropriate sections of the vehicle system has been exploited extensively in formulating crash simulation models in various attempts to simplify the modeling procedure and computation process [6-9].

Considering the unique severity posed by frontal crash which is neither opposed by the current interest in the demand and manufacture of highly economical cars of significantly reduced weight intended to meet certain requirements on environmental impact and drive energy economy, nor minimized by the persistent dependence on heavy 
vehicles for increased transportation needs; the study of impact energy absorption capacity and general crash performance of the front components of light-weight vehicles continues to represent an important area of research. A comprehensive review of relevant literatures reveals that the crash performance of vehicle structure in frontal impacts is largely dependent on the mechanical properties and the geometry of the front components. It is further noted that one elegant way to achieve a substantial theoretical report on this subject is to study the impact mechanics through appropriate LMS system capable of capturing the detailed nonlinear dynamics of the system. An objective review of the application of LMS method in vehicle crash simulation problems shows that the most reliable results could be traced to those in which the formulation of the dynamic resisting forces were based on the components' crush signature (force-deformation behavior). Such data are usually recorded for every major structural component under quasi-static condition provided by a low speed crusher [2]. However, the economic cost of implementing crush test is considerably high and discourages necessary investigation of the impact system in other comparable structural configuration(s) that may serve as a useful guide to structural designers and analysts in judging a good performance through such a method. In this regards detailed numerical model may be a good choice, though with relatively high demand on computation time. Consequently, illustration of desirable crash energy management system and other extended studies in vehicle impact problems that could be conveniently achieved through the LMS system approach or other reduced order dynamic models seems to rely on pure rudimentary procedures and unrealistic data capable of giving cursory assessments of the problem $[7,10]$. Considering the importance of the subject under study and the computational difficulty in achieving accurate results via the standard methods, researcher seek for possible ways to utilize the simplicity of LMS computation procedure to enhance illustration of the basic concept of crash energy absorption through structural deformation. Hamza [11] suggests a unique simulation method called the equivalent mechanism model which approximates the entire vehicle structure to a continuous chain of short rigid masses connected to each other via prismatic joints with nonlinear axial springs and revolute joints with torsional springs, and subsequently compared the results of the new method to that of an equivalent coarse finite element model. Other researchers attempted to simulate vehicle impact system via simplified linear visco-elastic one degree-of-freedom models [4,5]. Some others seek for further simplifications via equivalent square wave method [10], and multi-body model [12,13].

The observed trends stated above do not allow for proper appreciation of what may be considered good energy management system in a holistic sense due to lack of specific information on the effects of impact energy on the major energy absorbing members. The present study recognized the eight-component model structure first suggested by Kamal in his pioneering work [14] visualized in 4 DOF LMS system as a good framework capable of providing a comprehensive report on the crash performance of vehicle front structure. Critical assessment of such a simulation model reveals that the major energy absorbing components could be considered in reversed orientation in the system such that any valid data may be utilized in other structural configuration for comparative study without constituting any additional experimental cost. This idea is introduced in the present report for frontal impacts by observing the side rail in both normal orientation and reversed mode, assuming the other components to 
maintain their normal positions and geometry in the system. The expectation is to see the extent to which such a simple modification of structural configuration in the selected component could distinguish the good and the poor energy management system. The effort led to some important deductions which help in judging the impact system as observed in normal orientation as a better energy management system and emphasizes the need for proper characterization of front structures to ensure improved crash performance in light-weight vehicle crashworthiness design.

\section{Methods}

The research method involves state-space formulation of the vehicle crash energy management system based on the 4-DOF LMS model presented in Figure 1. The formulation of the model follows a well-known procedure for framed vehicle [2]. The assumed rigid masses represent the body mass $m_{1}$, the engine mass $m_{2}$, the cross- member and suspension mass $m_{3}$ and the bumper mass $m_{4}$. The resistances provided by the eight distinct energy absorbing sections identified in the vehicle system are represented as $F_{j}$; $j=1,2, \ldots, 8$. The torque box $F_{1}$ and the front frame $F_{2}$ constitute the side rail on which the suspension mass and the engine mass are supported. The resistance $F_{3}$ due to drive-line is recorded for a rear wheel drive, when the drive-shaft pushes rearwards and transmits impact load through the rear axle housing and rear-suspension into the frame. The resistance of the sheet metal (including the fender and other attached body above the frame and forward of the body) $F_{4}$ is separated by a physical clearance $C_{4}$ from the bumper mass. The firewall $F_{5}$ is the section that separates the engine with a suitable clearance $C_{5}$ from the rest of the body and prevents the engine mass from hitting the body mass in the early period of the impact. The resistance of the radiator unit (which includes the structures located directly in front of the engine behind the bumper mass) $F_{6}$ is equally located in the system with certain clearance labeled $C_{6}$ from the engine mass. The engine is supported by the side rail through a rubber mount with designated resistance $F_{7}$ attached to the torque box, and the transmission (gearbox) is fixed directly to the body through another rubber mount of resistance $F_{8}$.

The following simplifying assumptions are considered in arriving at the model.

i. Collision type is full-lap frontal impact against a rigid barrier.

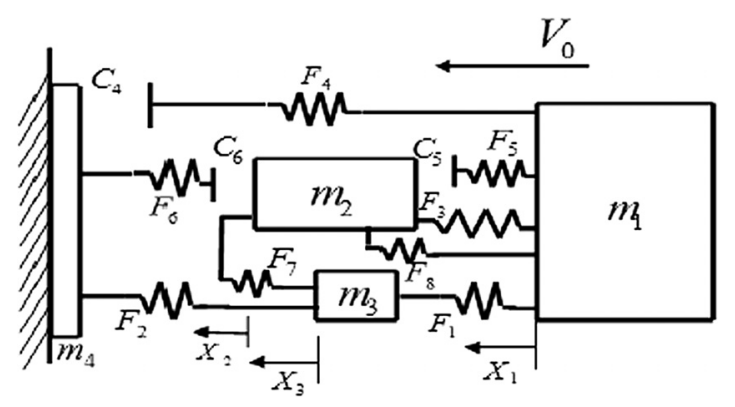

Figure 14 -DOF LMS model in normal orientation for evaluation of the front structure in crash energy management. 
ii. Sufficient rigidity is assumed in the passenger compartment to shield the occupant hence the body mass is lumped as $m_{1}$.

iii. The engine/transmission system or drive train (which include the gearbox, clutch system and drive shaft) and the cross-members/suspension system known to be structurally stronger than other sections are assumed un-deformable and collectively lumped as engine mass $m_{2}$.

iv. The resistances offered by the structural members forward of the engine mass against the barrier and that rearward of the engine mass against the body mass during the impact denoted $F_{j}$ correspond to the measured force deformation characteristics of the components.

v. The contributions to the resistance network due to highly flexible or fragile nonstructural members like cables, glasses, conduits, plastics etc. are considered negligible.

vi. The possible contribution of the structural masses to the inertia force vector is ignored.

\section{Equation of motion}

Considering the equilibrium of the 4-DOF force system (Figure 1), the motion of the system due to the impact is written as (1);

$$
\begin{gathered}
m_{1} \ddot{x}_{1}+F_{1}+F_{3}+F_{4}+F_{5}-F_{8}=0 \\
m_{2} \ddot{x}_{2}-F_{3}-F_{5}+F_{6}+F_{7}+F_{8}=0 \\
m_{3} \ddot{x}_{2}-F_{1}+F_{2}-F_{7}=0 \\
m_{4} \ddot{x}_{4}-F_{2}-F_{4}-F_{6}+F_{x}=0
\end{gathered}
$$

Rewriting (1) in compact form;

$$
M \ddot{X}+\sum F=0
$$

Where $\Sigma F$ is the matrix of the nonlinear resisting forces whose paths are mapped by linear force component $\alpha\left(F_{p}\right)$ in the elastic regime and a settling force $\phi\left(\delta, \dot{\delta}, F_{s}\right)$ in the plastic regime. The non-linear dynamics of the impact system is then explicitly written to reflect the possible transition from elastic state to a steady force state. This results in an equivalent non-homogeneous model (3);

$$
M \ddot{X}+\alpha\left(F_{p}\right)=\phi\left(\dot{\delta}, \delta, F_{s}\right)
$$

Considering the complex elastic-plastic motion of the system to be governed by inertia forces, linear spring resistance in the elastic regime and state dependent force in the plastic regime, then the gross motion of the system corresponds to the standard force balance (4)

$$
M \ddot{X}+K X=F(x, t)
$$

Where $M=m_{i j}$ is the matrix of the lumped masses $m_{i}$ and $X=x_{i}$ represents their position vector, $K=k_{i j}$ is the assembly of structural stiffnesses corresponding to elastic motion and $F(x, t)=f_{j}(\phi)$ in this construct, represents the vector of all internally generated forces in the spring set that sustains the plastic flow: $i=1,2, \ldots, n ; j=1,2 \ldots m$ for an $n \times$ $m$ mass-spring system. 
The state differential form of (4) utilized in the solution algorithm employs the transformation:

$$
\begin{aligned}
& x_{i}=\phi_{(2 i-1)}, \\
& \dot{\phi} k=\phi_{(k+1)} \text { for }: k=1,3, . .2 n-1 \\
& \dot{\phi}_{l}=\ddot{\phi}_{(l-1)} \text { for }: l=2,4 \ldots 2 n
\end{aligned}
$$

It then follows that for the 4-DOF model shown in Figure 1 the terms of the state differential equation can be defined in a reduced global coordinate system as:

$$
\begin{aligned}
M & =\left[\begin{array}{cccccccc}
1 & 0 & 0 & 0 & 0 & 0 & 0 & 0 \\
0 & m_{1} & 0 & 0 & 0 & 0 & 0 & 0 \\
0 & 0 & 1 & 0 & 0 & 0 & 0 & 0 \\
0 & 0 & 0 & m_{2} & 0 & 0 & 0 & 0 \\
0 & 0 & 0 & 0 & 1 & 0 & 0 & 0 \\
0 & 0 & 0 & 0 & 0 & m_{3} & 0 & 0 \\
0 & 0 & 0 & 0 & 0 & 0 & 1 & 0 \\
0 & 0 & 0 & 0 & 0 & 0 & 0 & m_{4}
\end{array}\right] ; \\
K & =\left[\begin{array}{cccccccc}
0 & 1 & 0 & 0 & 0 & 0 & 0 & 0 \\
\mathrm{k}_{21} & 0 & \mathrm{k}_{23} & 0 & k_{25} & 0 & \mathrm{k}_{27} & 0 \\
0 & 0 & 0 & 1 & 0 & 0 & 0 & 0 \\
\mathrm{k}_{41} & 0 & \mathrm{k}_{43} & 0 & \mathrm{k}_{45} & 0 & \mathrm{k}_{47} & 0 \\
0 & 0 & 0 & 0 & 0 & 1 & 0 & 0 \\
\mathrm{k}_{61} & 0 & \mathrm{k}_{63} & 0 & \mathrm{k}_{65} & 0 & \mathrm{k}_{67} & 0 \\
0 & 0 & 0 & 0 & 0 & 0 & 0 & 1 \\
\mathrm{k}_{81} & 0 & \mathrm{k}_{83} & 0 & \mathrm{k}_{85} & 0 & \mathrm{k}_{87} & 0
\end{array}\right] \\
\phi & =\left[\phi_{1}, \phi_{2}, \phi_{3}, \phi_{4}, \phi_{5}, \phi_{6}, \phi_{7}, \phi_{8}\right]^{\prime}, \dot{\phi}=\left[\dot{\phi}_{1}, \dot{\phi}_{2}, \dot{\phi}_{3}, \dot{\phi}_{4}, \dot{\phi}_{5}, \dot{\phi}_{6}, \dot{\phi}_{7}, \dot{\phi}_{8}\right]^{\prime}
\end{aligned}
$$

And

$$
f(\phi)=-\left[0, f_{1}(\phi), 0, f_{2}(\phi), 0, f_{3}(\phi), 0, f_{4}(\phi)\right]^{\prime},
$$

In compact notation the equation of motion in terms of the state variables is expressed as (10);

$$
M \dot{\phi}+K \phi=f(\phi)
$$

The state variables $\phi$ and $\dot{\phi}$ could be evaluated if the initial condition, stiffness matrix $K=k_{i j}$ and the vector of state dependent forces $f(\phi)$ are known or sufficiently characterized in the dynamic state.

Assuming full plastic collision against the rigid barrier and considering only the equilibrium of the partial elastic motion where $\delta_{p, j} \geq \delta_{j} \in x_{i}$, the contributions to the linear elastic forces $F_{p, j}\left(=k_{j} \delta_{p, j}\right)$ of the spring system $j=1,2, \ldots, 8$ are described in (11);

$$
\begin{aligned}
& F_{p, 1}=k_{1}\left(x_{1}-x_{3}\right) \equiv k_{1}\left(\phi_{1}-\phi_{5}\right), F_{p, 2}=k_{2} x_{3} \equiv k_{2} \phi_{5}, F_{p, 3}=k_{3}\left(x_{1}-x_{2}\right) \equiv k_{3}\left(\phi_{1}-\phi_{3}\right) \\
& F_{p, 4}=k_{4}\left(x_{1}-C_{4}\right) \equiv k_{4}\left(\phi_{1}-C_{4}\right), F_{p, 5}=k_{5}\left(x_{1}-x_{2}-C_{5}\right) \equiv k_{5}\left(\phi_{1}-\phi_{3}-C_{5}\right) \\
& F_{p, 6}=k_{6}\left(x_{2}-C_{6}\right) \equiv k_{6}\left(\phi_{3}-C_{6}\right), F_{p, 7}=k_{7}\left(x_{2}-x_{3}\right) \equiv k_{7}\left(\phi_{3}-\phi_{5}\right) \\
& F_{p, 8}=k_{8}\left(x_{2}-x_{1}\right) \equiv k_{8}\left(\phi_{3}-\phi_{1}\right)
\end{aligned}
$$

The net force controlling the motion of the individual masses $m_{i}$ is given by the vector $\sum_{j=1}^{m} F_{i, j}$, then comparing terms of the coefficient matrix $K$ resulting from the state space formulation (7) and its equivalent drawn from the linear map $\alpha\left(F_{p}\right)$ of the 
structural resistance in line with (1), (3) and (11), the unknown terms of the stiffness matrix $k_{i j}$ are written in terms of the specific elastic stiffness of the springs (12);

$$
\begin{aligned}
& \mathrm{k}_{21}=\left(k_{1}+k_{3}+k_{4}+k_{5}+k_{8}\right), \mathrm{k}_{23}=-\left(k_{3}+k_{5}+k_{8}\right), \mathrm{k}_{25}=-\mathrm{k}_{1}, \mathrm{k}_{27} \\
& =0, \mathrm{k}_{41}=-\left(k_{3}+k_{5}+k_{8}\right), \mathrm{k}_{43}=\left(k_{3}+k_{5}+k_{6}+k_{7}+k_{8}\right), \mathrm{k}_{45}=-\mathrm{k}_{7}, \mathrm{k}_{47}=0 ; \mathrm{k}_{61} \\
& =-\mathrm{k}_{1}, \mathrm{k}_{63}=-\mathrm{k}_{7}, \mathrm{k}_{65}=\left(\mathrm{k}_{1}+\mathrm{k}_{2}+\mathrm{k}_{7}\right), \mathrm{k}_{67}=0, \mathrm{k}_{81}=-\mathrm{k}_{4}, \mathrm{k}_{83}=-\left(\mathrm{k}_{2}+\mathrm{k}_{6}\right), \mathrm{k}_{85} \\
& =0, \mathrm{k}_{87}=0
\end{aligned}
$$

It is anticipated that, some components may not undergo significant plastic deformation due to high strength and the energy distribution pattern. The effect of the dynamic force vector $f_{i}(\phi)$ on the motion of the various masses is equally dependent on the number of components observed in full plastic state at the impact condition. The expression for $f_{i}(\phi)$ is therefore written to reflect these considerations in the form (13).

$$
f_{i}(\phi)=\sum_{\mathrm{r}} f_{i, \mathrm{r}}(\phi)
$$

Where $f_{i, \mathrm{r}}(\phi)$ represents the contribution to the total dynamic resistance about a specific mass $m_{i}$ due to a state dependent force generated in spring $\mathrm{r}$ found in plastic state.

\section{Load zone criterion}

Previous studies show that typical crush behavior of a nonlinear spring is illustrated by the load-deformation curve of Figure 2a which led to the identification of four (4) distinct load zones shown (zone 1-4). This knowledge is expanded in the present study in crucial effort to explain the true nature of the gross structural motion. An equivalent force-displacement diagram of Figure $2 \mathrm{~b}$ is drawn to account for possible instances of structural displacement without effective resistance in the system. In line with a standard LMS modeling procedure the total nonlinear force $F(x, t)$ that controls the impact motion is characterized by the load zoning formula (14) which accounts for the various forms identified by $F(x, t)$. from the initial state of the motion when a component is possibly sensing the impact without providing any significant resistance (zone Z0) through cases of stiff elastic motion (loading, unloading or reloading) against rigid wall (zone $\mathrm{Z1}$ ) and subsequent plastic flow (or localized buckling) under settling force (zones $Z 2$ and $Z 3$ ), up to the final case when the component becomes fully compressed, and transforms to solid mass. The transition from zone 3 orZ3 to zone 4 of the deformation-load path known as structural decomposition or total consumption by any component in the dynamic state is not obvious since the impact loads are readily

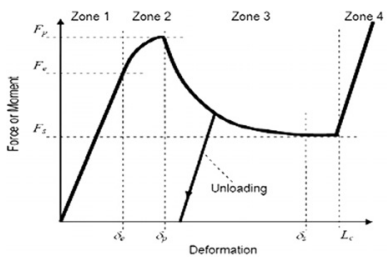

(a)

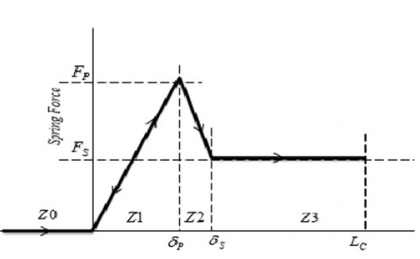

(b)

Figure 2 Illustration of; (a) the general component deformation pattern [7], and (b) the approximate force displacement diagram of the impact system. 
transmitted to the next compliant structure while the fully compressed component returns to zone Z0 and ensures sustained transmission of impact energy to the deforming components. It is noted that while a deforming component may traverse all or some the stated load zones depending on the nature/condition of the impact and structural configuration, there exists the possibility of the different components appearing in different load zones at certain instant. The dynamic resistance of a nonlinear spring system is then written for all possible load zones (14).

$$
\bar{F}(x, t)=\left\{\begin{array}{c}
\alpha\left(F_{p}\right) ; \text { for displacement in zone } Z 1 \text { or } 4 \\
\beta\left(\delta, F_{s}\right) ; \text { for displacement in zone } Z 2 \\
\psi\left(\dot{\delta}, F_{s}\right) ; \text { for displacement involving zones } Z 3 \\
0 ; \text { for displacement in zone } Z 0
\end{array}\right.
$$

Displacements found in zones Z1, Z2 and Z3 as indicated by the displacement-load path (Figure $2 \mathrm{~b}$ ) correspond to structural deformation and contribute substantially to energy absorption scheme; such zones are regarded in this paper as active load zones while zone $\mathrm{Z0}$, and zone 4 of the deformation load-path which lead to either insignificant energy absorption or total transmission of impact load are classified as idle (or passive) load zone. The transition from idle zone to active zone back to idle zone in addition to the switching of resistance formula and all other observed behaviors of the nonlinear springs were considered in arriving at the detailed governing equations of motion.

Further description of $\alpha\left(F_{p}\right), \beta\left(\delta, F_{s}\right)$, and $\psi\left(F_{s}, \dot{\delta}\right)$ which characterize the dynamic resistance at the corresponding load zones is given in Appendix section.

In the proposed method, the solution of the impact problem requires that the nonlinear spring geometric properties

$$
s_{j}=F_{p, j} / F_{s, j} ; p_{j}=\delta_{p, j} / L_{c, j} \text { and } q_{j}=\delta_{s, j} / L_{c, j}
$$

are first estimated under static condition from reliable force-deformation data, while the unknown dynamic parameters $F_{s, j}$ and $k_{j}$ of the governing state differential equation are then characterized preferably in terms of the specific energy absorption of the components under impact condition.

\section{Force deformation analysis}

The solution of the system response via the proposed method requires that the hysteretic parameter pair $\left(\mathrm{k}_{j}, F_{s, j}\right)$ which describes the load path of resistance must be quantified in the dynamic state. This implies that the contribution of every individual spring to the dynamic energy absorption sequence $\left(E_{j}\right)$ is known preferably as fraction of the total absorbable energy of the system $\lambda_{j}$. Hence, the proposed method adopts an approach in which all such contributions are matched in the dynamic state such that the solution of the system converges. The success of this approach lies on proper characterization of $E_{j}$ upon which the spring parameters $\mathrm{k}_{j}$ and $F_{s, j}$ are estimated. In the reviewed literatures $[1,2,7]$ the typical deformation behavior of the main energy members of front vehicle structures is illustrated by the generalized load-deformation curve of Figure 2a. In view of the complications and unmerited rise in computation time 
associated with tracing the details of the load path in the overall solution of $E_{j}$ in the proposed method, the reports are rather considered in a linearized form illustrated by the approximating force displacement diagram of Figure $2 \mathrm{~b}$ for developing the solution algorithm; assuming that a sufficient estimate of the force-deformation behavior and the energy absorption sequence could be achieved in the active load zones ( $\mathrm{Z} 1$, $\mathrm{Z} 2$ and Z3) via the approximate displacement model. This consideration enables detailed programing of the structural deformation sequence with minimized cases of iterative switches in the solution steps that essentially grants computational efficiency.

The individual energy absorption capacity of a component could be expressed as a fraction of the total absorbable energy according to the relation;

$$
E_{j}=\lambda_{j} E
$$

where

$$
E=\sum_{j=1}^{m} \lambda_{j} E+\varepsilon(T)=\left(\lambda_{1}+\lambda_{2}+, \ldots,+\lambda_{m}\right) E+\varepsilon(T)
$$

is the total absorbable energy.

Energy dissipation in form of heat, sound and vibration denoted by $\varepsilon(T)$ is usually assumed negligible so that all observed energy absorption in the system is credited to work done during structural deformation.

i.e

$$
\lambda_{1}+\lambda_{2}+, \ldots,+\lambda_{m} \cong 1
$$

To evaluate the spring parameter $F_{s, j}$ and $k_{j}$, the force-deformation characteristics of a given structural member (recorded either through static crush experiment or via equivalent numerical simulation) is first visualized in form of the approximate forcedisplacement behavior illustrated in Figure 2b. The spring geometric properties $s_{j}=F_{p, j} /$ $F_{s, j} ; p_{j}=\delta_{p, j} / L_{c, j}$ and $q_{j}=\delta_{s, j} / L_{c, j}$. (which characterize the contribution of individual component to the energy absorption scheme) are then evaluated.

Given the material balance;

$$
m=\sum m_{i}
$$

And energy conservation principle

$$
E=\sum E_{i}=0.5 \gamma m V_{0}^{2}
$$

The parameter $\gamma$ is a tolerance factor (or system adjustment variable) which could be used to tune the system to the best energy absorption performance during component design.

The individual energy absorption capacity $E_{j}$ of the springs is estimated by the area enclosed by the hysteretic force-displacement curve presented in Figure 2b, assuming that every displacement in the active load zone is equivalent to structural deformation.

$$
E_{j}=0.5 \delta_{s, j} F_{p, j}+F_{s, j}\left[\left(L_{c}-0.5\left(\delta_{s, j}+\delta_{p, j}\right)\right]\right.
$$

Equation 19 shows that the energy absorbing capacity of all nonlinear springs showing similar force-deformation characteristics varies according to the total crushable length $L_{c}$ of the components. The information may be useful at early design stage to 
enhance development of a workable design especially in integral body construction where vehicle front structures are usually made of intermediate columns of comparable deformation pattern.

Introducing the energy absorption index $\left(\lambda_{j}=E_{j} / E\right)$, then for a specific component $E_{j}$ is alternatively expressed as

$$
E_{j}=0.5 \gamma \lambda_{j} m V_{0}^{2}
$$

With the spring geometric properties $s_{j}=F_{p, j} / F_{s, j} ; p_{j}=\delta_{p, j} / L_{c, j}$ and $q_{j}=\delta_{s, j} / L_{c, j}$ estimated from a valid crush signature, the unknown spring parameters; peak force $F p_{j}$, mean (steady) force $F_{s, j}$, and the stiffness $k_{j}$ under crash condition are then readily obtained in terms of the specific energy absorption index $\lambda_{j}$.

$$
F_{s . j}=\frac{\gamma \lambda_{j} m V_{0}^{2}}{L_{c, j}\left[q_{j}\left(s_{j}-1\right)-p_{j}+2\right]}
$$

Since $F_{p, j}=s_{j} F_{s, j}$, the stiffness $k_{j}$ of a specific spring is then given

$$
k_{j}=\frac{F_{p, j}}{\delta_{p, j}}=\frac{\gamma \lambda_{j} s_{j} m V_{0}^{2}}{p_{j} L_{c, j}^{2}\left[q_{j}\left(s_{j}-1\right)-p_{j}+2\right]} \text { for } j=1,2, \ldots m
$$

The formulation of the dynamic steady-force $F_{s . j}$ and dynamic stiffness $k_{j}$. based on the specific energy absorption index and the known geometric properties of the components ensures convergence of the solution.

\section{Solution procedure}

To describe the detailed computation steps involved in evaluating the system response under dynamic impact condition the state differential form of the system is recalled in its standard format.

$$
\begin{aligned}
& \dot{\phi}_{1}=\phi_{2} \\
& \dot{\phi}_{2}=-\left[k_{21} \phi_{1}+k_{23} \phi_{3}+k_{25} \phi_{5}+k_{4} C_{4}+k_{5} C_{5}+f_{1}(\phi)\right] / m_{1} \\
& \dot{\phi}_{3}=\phi_{4} \\
& \dot{\phi}_{4}=-\left[k_{41} \phi_{1}+k_{43} \phi_{3}+k_{45} \phi_{5}-k_{5} C_{5}+k_{6} C_{6}+f_{2}(\phi)\right] / m_{2} \\
& \dot{\phi}_{5}=\phi_{6} \\
& \dot{\phi}_{4}=-\left[k_{61} \phi_{1}+k_{63} \phi_{3}+k_{65} \phi_{5}+f_{3}(\phi)\right] / m_{3} \\
& \dot{\phi}_{7}=\phi_{8} \\
& \dot{\phi}_{8}=-\left[k_{81} \phi_{1}+k_{83} \phi_{3}+k_{85} \phi_{5}-k_{4} C_{4}-k_{6} C_{6}-F_{x}+f_{4}(\phi)\right] / m_{4}
\end{aligned}
$$

The system response is computed dynamically based on the state variable formulation (23) subject to the load zone criteria (14). In the programming, the numerical integration utilizes various forms of Equation 23 in which each form reflects a unique observation of the spring system in the load zoning system. The number of independent observations utilized in the programing was minimized based on some practical considerations. Only components that show both linear elasticity and significant plasticity in the static crush characteristics data were considered in both perspectives in the solution program. For practical details additional cases were recognized for pure dynamically compliant springs in fully compressed and totally consumed states so as to improve the accuracy of the current method at impact speed of about $50 \mathrm{~km} / \mathrm{h}$ where extended structural deformation is anticipated. By and large, crash modeling is usually 
intended to minimize fatality in a survivable crash occurring at moderate impact velocity range usually below $100 \mathrm{~km} / \mathrm{h}$. At this range, transition to fully compressed or totally consumed state by the interior components of real vehicle structural rigidity is certainly not desirable.

Considering separately the two structural modes under study at full-compliant state (where $\gamma=1$ ), the distinct energy absorption capacities $E_{j}$ of the nonlinear springs were first evaluated from Equations 20 for a specific value of energy absorption index $\lambda_{j}$. This enables the calculation of dynamic parameters $F_{s, j}$ and $k_{j}$ from (21) and (22) respectively, substituting the known components' geometric properties $p_{j}, q_{j}, s_{j}$, and $L_{C, j}$ (i.e. measured force-deformation behavior for a typical framed car components found in $[1,2]$ mapped as proposed in Figure 2b), and the typical mass distribution of a conventional light-vehicle given in Appendix section. The results of this first stage analysis known as spring tuning were then applied for the solution of the system response under crash condition through a computer program written to solve the governing differential equations of motion for a specific value of $\lambda_{j}$ (accounting for all observed cases of mass displacements and structural load zones) given the initial conditions $\left(\dot{\mathrm{X}}_{1}=\dot{\mathrm{X}}_{2}=\dot{\mathrm{X}}_{3}=\dot{\mathrm{X}}_{4}=\right.$ $\left.V_{0}, x_{1}=x_{2}=x_{3}=x_{4}=0\right)$. The solutions were completed through numerical integration employing simple logics that check the deformation states of the springs and select appropriate governing differential equation corresponding to each case such that the displacements and velocities of the masses arising from a preceding case are automatically fed as initial conditions to the new governing equation in the current case.

\section{Results and discussion}

The present research problem was solved based on state variable formulation. The resulting accelerations of the various masses were integrated iteratively using ODE45 numerical solver. The results are presented as time histories of the impact events. The necessary comparison between the results of the impact system in normal and the reversed structural modes were recorded accordingly. As anticipated some significant disparities were observed in all the compared events. The results obtained in terms of displacement, velocity and acceleration of the masses are all typical of compliant vehicle structures that show significant plastic deformation in both the foremost structures and the interior front components.

\section{Displacement response}

Observation of the displacement responses of the lumped masses in the reversed structural mode (Figure $3 \mathrm{~b}$ ) relative to the normal operation mode (Figure 3a) shows dramatic reduction in the peak displacement and early rebound of the suspension mass, accompanied by some marginal increase in the displacements of the body and the engine masses with significant rebound seen towards the end of the impact. This is consequent to the reduction of the total crushable length of the front frame achieved in the reversed structural mode. In the normal mode, the various masses excluding the bumper mass show almost equal amount of instantaneous displacement with the body mass showing slightly higher peak value due to increased inertia force. The bumper mass is stuck to the rigid barrier all through the duration of impact, showing no 


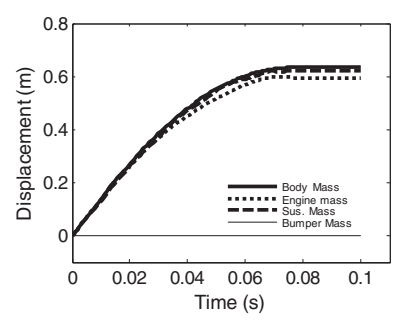

(a)

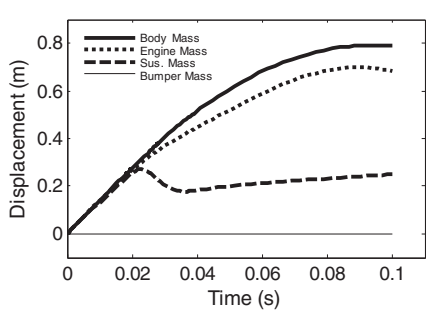

(b)

Figure 3 Displacement history of the impact system in normal mode (a) and reversed mode (b).

noticeable displacement but ensuring sustained transmission of the impact energy to the collapsing structures.

\section{Velocity response}

The velocity responses gathered for the various displaced masses in the normal operation mode in Figure 4a reflect what may be desirable in crash energy management campaign. The masses show uniform and complete loss of the initial impact velocity well within the impact duration, with the velocity profiles interlocking each other at some instances, recording only certain negligible restitution towards the end of the impact. By and large, the suspension mass depicts more frequent oscillation in the velocity history due to its small mass value. In the reversed structural mode (Figure $4 \mathrm{~b}$ ), the resulting unreasonably high deceleration of the suspension mass caused by rapid consumption of the front frame forced its velocity path to separate significantly from those of the associated masses, showing excessively high restitution at the early impact stage. The resulting poor energy management plan also led to significant restitution of the engine and the body masses towards the end of the impact event suggesting increasing fatality.

\section{Acceleration response of the body mass}

Further comparison of the system response was accomplished through the recorded peak acceleration/deceleration of the body mass. Observing the results at each iteration steps shows that operating the system in normal and reversed structural configurations results in comparable peak body mass decelerations of $31.5 \mathrm{~g}$ and $30.7 \mathrm{~g}$ respectively. This occurred in both cases at the instant when the front frame and the sheet metal were in steady force state. Both figures fall within acceptable range recommended by

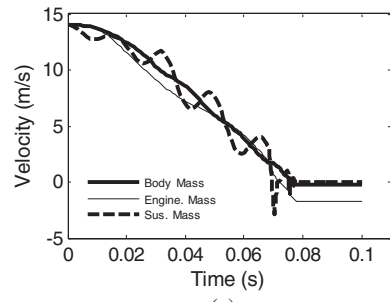

(a)

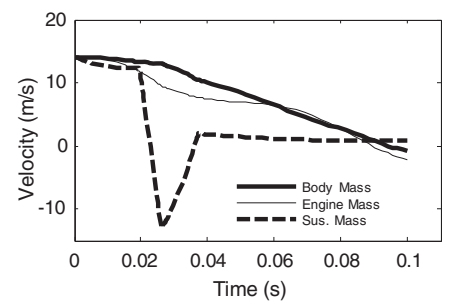

(b)

Figure 4 Velocity response of the impact system in normal mode (a) and reversed mode (b). 
automobile safety regulatory bodies [10]. Hence further assessments of the relative performance of the exemplified structural plans in impact energy management were embarked upon using the measured instantaneous axial crush of components and the total energy loss history as follows.

\section{Axial crush of components}

The deformation histories of the distinct energy absorbing members that record substantial axial crush are presented in comparable instances. In the normal structural mode (Figure 5a), high component axial crush is restricted to the foremost structures including front frame $\left(F_{2}\right)$, radiator unit $\left(F_{6}\right)$, and sheet metal $\left(F_{4}\right)$ which are finally found in compressed state between the barrier and the engine mass. The interior structures are rather locked in some form of uncertain deformation style that resulted in very little compression of the torque box $\left(F_{1}\right)$, the drive-line $\left(F_{3}\right)$, and the firewall $\left(F_{5}\right)$, while the engine and the transmission rubber mounts $\left(\mathrm{F}_{7}\right.$ and $\left.\mathrm{F}_{8}\right)$, which are capable of resisting in both forward and rearward directions experience only slight extensions (Figure 6a). The front frame and the sheet metal show approximately equal amount of instantaneous axial crush which increases exponentially from zero in the beginning of the impact and eventually settles at the maximum crushable length of the front frame $(0.62 \mathrm{~m})$. A slightly reduced peak axial crush is measured in the radiator unit due to the observed physical clearance that separates the unit from the engine mass and prevents early crushing of the radiator unit. The overall outcome supports minimized intrusion of the deforming interior structures and of course the un-modeled sub-components (like the steering column, dashboard structures, brake and other control levers) into the passenger compartment suggesting a good impact energy management system.

On the other hand, the reversed system witnessed rapid consumption of the front frame within the first 20 milliseconds of the impact leading to transfer of huge amount of the impact load on the other components. As a result both the radiator and the sheet metal experienced unreasonably high peak deformation. Some of the interior structures which include; the torque box and the engine mount equally show extended deformation, suggesting increased structural intrusion and poor energy management scheme (Figure 5b). However, the firewall, the drive-line and transmission mount maintain similar deformation pattern as in normal structural mode but with slightly increased peak values (Figure 6b).

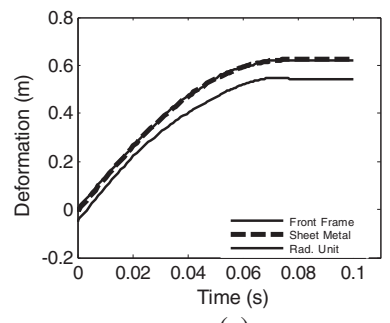

(a)

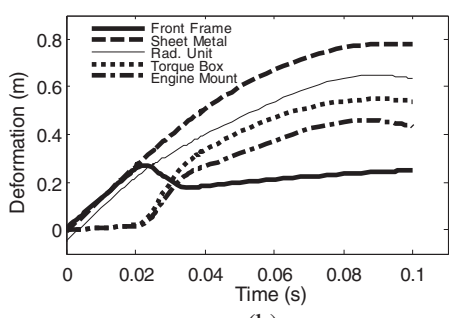

(b)

Figure 5 Axial crush record of the highly deformed front components of the impact system in normal mode (a) and reversed mode (b). 


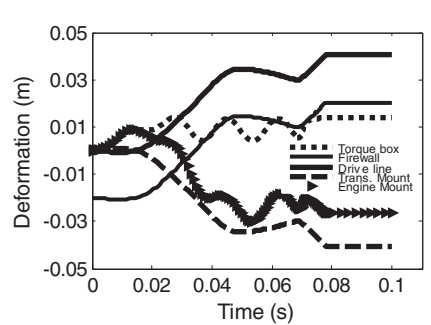

(a)

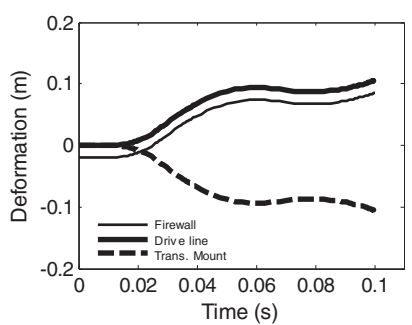

(b)

Figure 6 Axial crush record of the less deformed interior components of the impact system in normal mode (a) and reversed mode (b).

\section{Energy absorption}

The validity of the present study is demonstrated by the records of the energy loss history presented in Figure 7. The results are typical of a considerably inelastic collision. All the initial kinetic energy of the system eventually reduced to zero towards the end of the impact in the two case studies. However, Figure 7a depicts a better energy management system since the desire to absorb the whole impact energy through structural deformation is met well within the impact duration following a smooth sloping work rate.

The assessment of individual contributions of the various components to the energy absorption sequence is based on the resulting energy absorption indices (Table 1) recorded as the solution converges. These results represent possible absorption of the impact energy in the various structural zones. It is noted that in the normal structural mode, the major contribution to the energy absorption scheme is through the deformation of the foremost structures; the front frame (45\%) and the sheet metal (25\%). This trend in energy absorption is responsible for the considerably large total axial crush recorded by these components as shown in Figure 5a. The transmission mount and the torque box contribute up to $10 \%$ and $20 \%$ of the total energy absorption respectively, while the other interior members show very small contributions. The absorption of greater fraction of the total impact energy through extended deformation of the foremost structures associated with the normal mode reflects what is desirable in crash energy management campaign since it supports low energy absorption by the interior components and minimized structural intrusion into the passenger compartment. The result obtained for the reversed structural mode indicates a rather poor energy

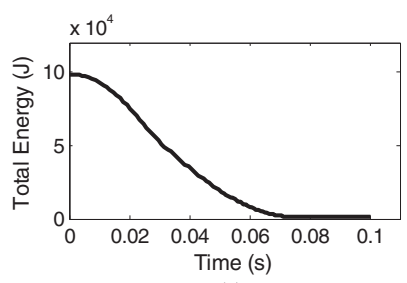

(a)

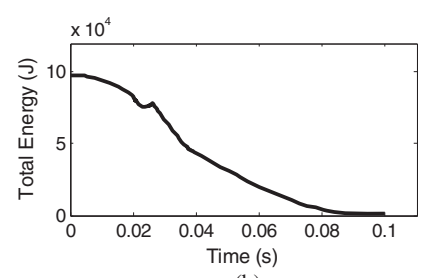

(b)

Figure 7 Instantaneous energy profile of the impact system in normal mode (a) and reversed mode (b). 
Table 1 Specific energy absorption indices of the structural components

\begin{tabular}{lllllllll}
\hline Structural mode & $\boldsymbol{\lambda}_{\mathbf{1}}$ & $\boldsymbol{\lambda}_{\mathbf{2}}$ & $\boldsymbol{\lambda}_{\mathbf{3}}$ & $\boldsymbol{\lambda}_{\mathbf{4}}$ & $\boldsymbol{\lambda}_{\mathbf{5}}$ & $\boldsymbol{\lambda}_{\mathbf{6}}$ & $\boldsymbol{\lambda}_{\mathbf{7}}$ & $\boldsymbol{\lambda}_{\mathbf{8}}$ \\
\hline Normal & 0.20 & 0.45 & 0.001 & 0.25 & 0.001 & 0.015 & 0.001 & 0.10 \\
Reversed & 0.43 & 0.025 & 0.001 & 0.50 & 0.001 & 0.010 & 0.001 & 0.05 \\
\hline
\end{tabular}

absorption pattern in which unreasonably high percentage (43\%) of the total impact energy is absorbed through the deformation of the interior torque box only. This caused an incredible amount (50\%) of the total impact energy to be absorbed through extreme deformation of the sheet metal. The resulting trend implies more risk of structural intrusion into the passenger compartment and increasing fatality.

\section{Future work}

Considering the cost of obtaining full-scaled crash test data, the development of a workable dynamic vehicle crash model based on static crush behavior of the structural components conceived in this study represents a significant simplification in vehicle crash design capable of enhancing on-line decision during design for vehicle structural crashworthiness. The proposed method quantifies the contributions of the various component in impact energy absorption by observing the effects $\lambda_{j}$ on the system response within a useful range of values such that the known initial and of course the anticipated final conditions of the problem are substantially realized. Fast convergence of the solution is always guaranteed since the sampling of $\lambda_{j}$ is conducted within a short data range of $(0-1)$. The solution converges when the velocity of the system (or the bumper force) approaches zero within typical impact duration assuming full-plastic collision. Further studies may be tailored to validate the proposed computation method through standard numerical method such as finite element method. Recent developments in CAD/CAE increase the possibility of obtaining reliable component static force-deformation behavior through numerical simulation once the mechanical properties and geometry of the components are specified. It then implies that the expected crash behavior of any proposed design could be readily tested during component formulation, allowing for prompt system adjustment in the case of any observed indication of unwanted performance, even before final prototype assembly using the proposed method. This represents a significant cost reduction from conducting multiple real component crush test or full-scaled crash test experiments and computation time saving from repeated detailed numerical simulation of fully assembled vehicle model needed during component formulation. With the crush characteristics of components attained through standard numerical simulation method, the necessary validation of the proposed modeling procedure could be achieved through straightforward comparison of the results of the proposed simulation of vehicle crash response via integrated LMS model employing component static force deformation behavior and those of an equivalent fully assembled system attained through detailed finite element method. Other necessary extension of the present work may be directed towards developing automated system for monitoring the solution convergence (which may require the use of graphical/numerical optimization tool such as genetic algorithm or differential evolution) that would generally enhance the application of the proposed method in crash performance evaluation. 


\section{Conclusion}

The present study reveals that proper design for light-weight vehicle that shows high crashworthiness potential in frontal impact such as; minimized intrusion of structures into the passenger compartment, controlled restitution/rebound of the vehicle masses, acceptable occupants' acceleration and maximum absorption of impact energy via structural deformation could be achieved through adequate evaluation of the vehicle front components in crash energy management system using the proposed method. The results of the present study suggest that the design criteria on frontal impact in terms of standard upper limit of acceleration of the occupants (or payload mass) of $32 \mathrm{~g}$ and minimized intrusion of structure into the passenger compartment, could be realized by ensuring sufficiently high $(\geq 65 \%)$ contribution to the energy absorption scheme through the deformation of the front frame and the sheet metal. As seen from the results of the normal structural mode, this amount of energy absorption ensures that significantly reduced fractions of the total impact energy are absorbed by the interior front structures. Moreover, the studied crash energy management plan represented as the normal structural mode grants fully resisted, unidirectional (no rebound) displacement, minimal terminal restitution and fairly uniform deceleration of the vehicle masses which are all desirable crash trends. The necessary design considerations for reaching such a desired crash performance involve proper selection of structural stiffness and component geometry which determine the distribution of impact energy in the vehicle system. It is noted that the dynamic resistance of the vehicle structures and the associated energy absorption during the impact depend on such distribution of the impact energy within the structural zones. Hence, a major contribution of this work is the construction of the dynamic peak/mean resistant forces and the dynamic structural stiffness based on specific energy absorption of the main structural members which enables the evaluation of the unknown dynamic resistance and the system crash response. The demonstrated efficiency of the proposed method represents significant relief from the usual computational burden presented by the existing methods.

\section{Appendix}

Definition of spring parameters:

The total dynamic resistance of the structural system is given as sum of the partial resistance corresponding to the three identified active load zones (A1).

$$
F_{j}=\operatorname{sig} \alpha\left(F_{p, j}\right)+\operatorname{sig} \beta\left(\delta_{j}, F_{s, j}\right)+\operatorname{sig} \psi\left(F_{s, j}, \dot{\delta}_{j}\right)
$$

The resistance is further described in active load zones; Z1, Z2 and Z3 as follows

$$
\begin{aligned}
F_{j, 1} & =k_{j} \delta_{j} \\
F_{j, 2} & =F_{s, j}(v+\xi \delta)_{j} ; \text { Where } v=\frac{q s-p}{q-p} ; \xi=\frac{1-s}{L(q-p)} \\
F_{j, 3} & =F_{s, j}
\end{aligned}
$$

The entire load path of resistance is hence described by (A3) (Tables 2 and 3)

$$
\begin{aligned}
& \alpha\left(F_{p}\right)=k_{i j} \delta_{p, j} ; \\
& \beta\left(\delta_{j}, F_{s, j}\right)=\operatorname{sign} \dot{\delta}_{j} f_{j, 2} \\
& \psi\left(F_{s, j}, \dot{\delta}_{j}\right)=\operatorname{sign} \dot{\delta}_{j} f_{j, 3}
\end{aligned}
$$


Table 2 Estimate of static force deformation properties of the various components in both the normal and the reversed structural modes obtained from crush test data [2]

\begin{tabular}{|c|c|c|c|c|c|c|c|c|c|}
\hline$j$ & $L_{c, j}$ & $L_{c, j, R}$ & $p_{j}$ & $p_{j, R}$ & $q_{j}$ & $q_{j, R^{*}}$ & $s_{j}$. & $s_{j, R}$ & $C_{j}$ \\
\hline 1 & 0.25 & 0.62 & 0.06 & 0.50 & 0.47 & 0.61 & 2.0 & 1.3 & - \\
\hline 2 & 0.62 & 0.25 & 0.50 & 0.06 & 0.61 & 0.47 & 1.3 & 2.0 & - \\
\hline 3 & 0.25 & 0.25 & 0.30 & 0.30 & 1.00 & 1.00 & - & - & - \\
\hline 4 & 1.50 & 1.50 & 0.25 & 0.25 & 0.40 & 0.40 & 2.5 & 2.5 & 0.01 \\
\hline 5 & 0.50 & 0.5 & 1.00 & 1.00 & - & - & - & - & 0.02 \\
\hline 6 & 0.11 & 0.11 & 1.00 & 1.00 & - & & & & 0.05 \\
\hline 7 & 0.11 & 0.11 & 1.00 & 1.00 & - & - & - & - & - \\
\hline 8 & 0.10 & 0.10 & 0.54 & 0.54 & 0.67 & 0.67 & 1.0 & 1.0 & - \\
\hline
\end{tabular}

$$
\operatorname{sign}(x)=\left\{\begin{array}{c}
-1 ; x<1 \\
0 ; x=0 \\
1 ; x>1
\end{array}\right.
$$

Abbreviations

CAD, Computer aided design

CAE, Computer aided engineering

DOF, Degree of freedom

LMS, Lumped mass-spring system

RODM, Reduced order dynamic model

FEM, Finite element method

Symbols

$K$ Stiffness matrix

$C$ Clearance space

$\delta_{p, j}$ Peak displacement of individual spring $j$ corresponding to linear elastic loading $\delta_{s, j}$ Minimum axial crush of individual spring $j$ corresponding to steady force zone

$E$ Total absorbable energy

$E_{j}$ Energy absorption capacity of a specific nonlinear spring $j$

$F_{j}$ Nonlinear resistant force generated in spring $j$

$F_{s, j}$ Steady force associated with plastic deformation of spring $j$

$F_{p, j}$ Peak force associated with elastic deflection of spring $j$

$f_{i j}(\dot{\delta})$ Contribution to the dynamic resistance of a specific mass $i$ due to a steady force generated in a member $j$

$F_{j, h}$ Dynamic resistant force generated in zone $Z h: h=1,2,3$

$F(x, t)$ A state dependent force

Table 3 Distributed masses of vehicle sections utilized in the solution showing the initial conditions

\begin{tabular}{llll}
\hline $\boldsymbol{i}$ & $\dot{\mathrm{x}}_{\mathbf{0}, \boldsymbol{i}}(\mathbf{m} / \mathbf{s})$ & $\boldsymbol{x}_{\mathbf{0}, \mathbf{i}}$ & $\boldsymbol{m}_{\boldsymbol{i}}(\mathbf{k g})$ \\
\hline 1 & 14 & 0 & 750 \\
2 & 14 & 0 & 170 \\
3 & 14 & 0 & 70 \\
4 & 14 & 0 & 10 \\
\hline
\end{tabular}


$\bar{F}(x, t)$ Generalized nonlinear resistant force for all identified load zones

$\mathrm{k}_{j}$, Linear stiffness of a specific component (nonlinear spring) $j$

$L_{c}$ Total crushable length of component

$M$ Mass matrix

$m$ Mass

$p$ Ratio of peak deformation to total crushable length

$q$ Ratio of minimum deformation in steady force zone to total crushable length

$s$ Peak force to steady force ratio

$\lambda$ Absorbable energy index (specific energy absorption)

$\alpha(F p)$ Coordinate of the peak force vector

$\phi(\delta, \dot{\delta}, F s)$ Coordinate of steady settling force vector

$X$ Position vector

$\ddot{X}$ Acceleration vector

Units

N Newton

Competing interests

The authors declare that they have no competing interests.

\section{Authors' contributions}

OSM carried out the research on crash performance evaluation of vehicle front structure, compiled the results and drafted the manuscript. OCG developed the research methodology and assisted in some computation work. ESO supplied the basic programming resources and assisted in the actual programming of the overall computation procedure. All authors read and approved the final manuscript.

\section{Author details}

${ }^{1}$ Department of Mechanical Engineering, Nnamdi Azikiwe University, Awka, Nigeria. ${ }^{2}$ Department of Mechanical Engineering, University of Nigeria, Nsukka, Nigeria.

Received: 6 October 2014 Accepted: 2 January 2015

Published online: 01 April 2015

\section{References}

1. Augustitus JA, Lin K, Kamal M (1975) Computer simulation of vehicle-to-barrier impact -A user guide, General Motors Research Publication, GMR-1943

2. Balike M (1998) Enhancement of Crashworthiness in Car-Truck Collisions Using Damped Under-Ride Guard, Doctoral Degree Research. Department Of Mech. Engineering, Concordia University, Montreal

3. Robbersmyr KG (2004) Calibration Test of a Standard Ford Fiesta 1.1 I, Model Year 1987, according to NS - EN 12767, Technical Report 43/2004, Agder University College, Grimstad

4. Karimi HR, Robbersmyr KG, Pawlus W (2011) Development of lumped-parameter mathematical models for a vehicle localized impact. J Mech Sci Technol 25(7):1737-1747

5. Karimi HR, Robbersmyr KG, Pawlus W (2011) Mathematical modeling and parameters estimation of a car crash using data-based regressive model approach. Appl Math Model 35:5091-5107

6. Alexandra C, Stuart GM, Samaha RR (1995) "Lumped parameter modeling of frontal offset impacts", SAE paper 950651

7. Witteman WJ. Improved vehicle crashworthiness design by control of the energy absorption for different collision situations, Ph.D. thesis, Eindhoven University of Technology, Eindhoven, The Netherlands, ISBN 90-386-0880-2, 1999

8. Karimi HR, Robbersmyr KG, Pawlus W (2010) Mathematical modeling of a vehicle crash test based on elasto-plastic unloading scenarios of spring-mass models. Int J Adv Manuf Technol. DOI 10.1007/s00170-010-3056-x

9. Marzbanrad J, Pahlavani M (2011) Paremaeter Determination of a Vehicle 5-DOF Model to Simulate Occupant Deceleration in a Frontal Crash, World Academy of Science, Engineering and Technology 55

10. King Al, Fileta BB, Chou CC, Mahmood HF, Mertz HJ, Wismans J, Bois PD, Khalil TB (2004) Vehicle Crashworthiness and Occupant Protection. American Iron and Steel Institute 2000 Town Center Southfield, Michigan, p 48075

11. Hamza K, Saitou K (2005) Design for structural crashworthiness using equivalent mechanism approximations. J Mech Des 127:485-492

12. Pennestrì E, Vita L, Valentini PP (2005) Comfort analysis of car occupants: comparison between multi-body and finite element models. Int J Vehicle Syst Model Test 1:1/2/3

13. Peng C, Chang F, Teng T, Liu Y (2008) Analysis of dynamic response of vehicle occupant in frontal crash using multi-body dynamics method. Math Comput Model 48:1724-1736

14. Kamal MM (1970) Analysis and Simulation of Vehicle to Barrier Impact, International Automobile Safety Conference, Detroit Michigan, May 13-17, SAE paper No. 700414., p 197 